\title{
Method for analysing the adequacy of electric power systems with wind power plants and energy storages
}

\author{
Sergey Perzhabinsky ${ }^{1 *}$, Dmitriy Karamov $^{1}$ \\ ${ }^{1}$ Melentiev Energy Systems Institute, 130 Lermontov str., Irkutsk, Russia
}

\begin{abstract}
Currently, renewable energy sources and energy storage devices are actively introduced into electric power systems. We developed method to analyze the adequacy of these electric power systems. The method takes into account the uncertainty of electricity generation by wind power plants and the processes of energy storage. The method is based on the Monte Carlo method and allowed to use of long-term meteorological data in open access. The performed experimental research of electrical power system is constructed on the basis of the real technical and meteorological data. The method allows to estimate of effectiveness of introducing generators based on renewable energy sources and energy storages in electric power systems.
\end{abstract}

\section{Introduction}

The modern development of electric power systems (EPS) is characterized by a significant increase in generating capacity with renewable energy sources (RES). Necessary highlights the use of RES in decentralized power systems of medium and low power levels, where both photovoltaics (PV) and wind turbines (WT) have become very popular. At the same time, Russian and foreign experience shows that the use of PV, WT and energy storages (ES) is an economically efficient way of energy supply to consumers. For example, in the Far East Federal District, there are fifteen small-scale solar power plants (from 10 to 100 $\mathrm{kW}$ ) which are located in decentralized power systems. The larger projects carried out in the Far East Federal District use wind power. Starting from 2013 to the present time, three projects have been implemented, that using wind turbines ( $250 \mathrm{~kW}$ to $1100 \mathrm{~kW}$ ).

It is worth noting that the generation of WT and PV has a stochastic, oscillating character. Of course, in such conditions, justified to use energy storages (ES) acting as a stabilizing element of a system operating in a buffer mode. In addition to a pronounced stabilizing effect, the presence of ES significantly improves the reliability of power supply, due to the availability of additional storage capacity, which allows to eliminate energy deficit caused by emergency shutdowns.

Possible approaches to the analysis of balance reliability of EPS are discussed in a number of works [13]. In [4], an effective methodology for estimating the balance reliability of EPS, based on the Monte-Carlo method, is presented. According to this methodology, at the first stage calculation of values of random variables (maximum possible generating capacity, power handling capacity of overhead line, electrical load) are simulated that characterize some operating state of the EPS. Then, ES power deficit is estimated, and reliability indicators are calculated (probability and mathematical expectation of undersupply of energy). This methodology has proven itself in the long-term planning of EPS and was implemented in the software. Based of this methodology have been developed algorithms to optimize reliability of EPS $[6,7]$.

To adapt the methodology to modern conditions, it is necessary to take into account the following provisions: accidental generation of energy of WT; modification the model to assess expectation of undersupply of energy with taking into consideration energy storage system in electrical power systems; take into consideration the charge-discharge processes of ES, with considering a number of operational and technical boundary conditions; to develop reliability indicators reflecting the availability of new types of equipment in electric power systems.

In [8], methods for simulating wind activity are presented and a modification of the methodology is given for analyzing the reliability of EPS with wind farms without energy storage system. In the literature, the task of analyzing the balance reliability of EPS with WT and energy storage system has been studied in detail [9-10]. It is worth noting that in [9] advantages are shown for using ES in electric power systems with WT. However, the reliability of the distribution network elements is not taken into account (overhead line, power electronics, etc.) of high and medium level of voltage, which undoubtedly affects the results. Also, in [9-10], clearly do not take into consideration mode indicators of ES, types of used ES, load factor in the moments shortterm disturbances with the observance of a number of technical limitations and the period of their lifetime. 
A number of approaches to modeling of wind activity and modeling of appropriate models of reliability assessment are suggested: analysis of the data of longterm observations of the wind speed of the territory under consideration and used of the mathematical expectation of the hourly wind speed [11]; construction of autoregressive models of wind speed [12]; application of the Monte Carlo method according to the scheme of Markov chains [13]; use of a hybrid algorithm of machine learning based on wavelet transform and artificial neural network [14], etc.

The goal of this article is to develop a methodology for analyzing the reliability of EPS with WT and ES. In the first section of this article, estimation model a modification of the undersupply of energy with quadratic power losses in overhead line is considered. Theoretical studies of the model with quadratic power losses, presented in [16], indicate the effectiveness of using this model in analyzing the reliability of EPS. The second section of this article discusses the features of modeling random variables and calculating reliability indicators. The third section is devoted to the description of the results of the computational experiment and the conclusions.

\section{The model of power shortage estimation of electric power systems with energy storages}

Let's consider a scheme of electric power system which contains $\mathrm{n}$ nodes and set of system links. The given power system contains energy storages. Let $\mathrm{N}$ is number of simulated states of electric power system. Each system state is characterized of set of random values such as of available generating capacity $\bar{x}_{i}^{k}$, value of load $\bar{y}_{i}^{k}$ in node $\mathrm{i}$, power line capacity $\bar{z}_{i j}^{k}$ between nodes $\mathrm{i}$ and $\mathrm{j}, \quad i=1, \ldots, n, \quad j=1, \ldots, n, \quad i \neq j$, $k=1, \ldots, N$. Maximal $\overline{\Delta x i}$ axax minimal $\overline{\Delta x i}$ min capacities of storage are given for each node i. Current power $\underline{\Delta x}_{i}^{k}$ and available capacity of energy storage $\overline{\Delta x i}$ are computed on the base of characteristics of previous $k-1$ state of energy storage, $k=1, \ldots, N$.

Let $x_{i}$ is power used at the node $\mathrm{i}, y_{i}$ is the power served at the node i, $\Delta x_{i}$ is variation of battery power condition at $\mathrm{i}$-th energy storage, $z_{i j}$ is power flow from the node $\mathrm{i}$ to the node $\mathrm{j}, i=1, \ldots, n, j=1, \ldots, n$, $i \neq j$. We propose following new problem for power shortage estimation of system state with the number $\mathrm{k}$, $k=1, \ldots, N$.

$$
G \sum_{i=1}^{n} y_{i}+\sum_{i=1}^{n} \Delta x_{i} \rightarrow \max ,
$$

subject to constraints

$$
\begin{gathered}
x_{i}-y_{i}+\sum_{j=1}^{n}\left(1-a_{j i} z_{j i}\right) z_{j i}-\sum_{j=1}^{n} z_{i j}-\Delta x_{i}=0, i=1, \ldots, n,(2) \\
0 \leq y_{i} \leq \bar{y}_{i}^{k}, i=1, \ldots, n, \\
0 \leq x_{i} \leq \bar{x}_{i}^{k}, i=1, \ldots, n, \\
-\underline{\Delta x_{i}^{k}} \leq \Delta x_{i} \leq \overline{\Delta x} i, i=1, \ldots, n, \\
0 \leq z_{i j} \leq \bar{z}_{i j}^{k}, i=1, \ldots, n, j=1, \ldots, n, i \neq j .
\end{gathered}
$$

Here $a_{i j}$ are given positive coefficients of specific power losses during electric energy transfer from the node $\mathrm{i}$ to the node $\mathrm{j}, i \neq j, \mathrm{G}$ is weight number for raising of priority of minimization of power deficit, $G \gg>1, \quad i=1, \ldots, n, j=1, \ldots, n$. Variables $\Delta x_{i}$, $i=1, \ldots, n$, can be negative. It means that load in system is bigger than generating power and requisite power is consumed from energy storage.

Let $\Delta x_{i}^{k-1}$ are optimal values that are got as a result of solving problem (1)-(6) for EPS state with number $k-1, i=1, \ldots, n, k=1, \ldots, N$. Values $\underline{\Delta x}_{i}^{k}$ and $\overline{\Delta x} i$ , $i=1, \ldots, n, k=2, \ldots, N$, are recurrently computed by formulas

$\underline{\Delta x}_{i}^{k}=\underline{\Delta x}_{i}^{k-1}+\Delta x_{i}^{k-1}, \overline{\Delta x} k=\overline{\Delta x}_{i}^{\max }-\underline{\Delta x}_{i}^{k}$.

For the computing $\underline{\Delta x}_{i}^{0}$ you can use any meaning of random value $\zeta$ which is equally spaced on the interval $\left[\overline{\Delta x i}^{\min }, \overline{\Delta x i}^{\max }\right]$.

The process of charging and consumption of energy from energy storage can be presented in a view as Markov chain [15]. Applying of described approach is allowed to simplify a modelling of process of energy storage's work. We can consider a meaning of random values $\underline{\Delta x}_{i}^{k}, \overline{\Delta x} k$ as equally probable at the cost of big number of EPS states.

A solution of the problem (1) - (6) is exist because the feasible set of the problem is limited and not empty (solution $y_{i}=0, x_{i}=0, \Delta x_{i}=0, z_{i j}=0, i=1, \ldots, n$, $j=1, \ldots, n, i \neq j$ is a feasible) and objective function is linear. The uniqueness of optimal solution of the problem (1)-(6) by variables $y_{i}, i=1, \ldots, n$, follows from proved theorems in [1]. The next theorem is true. Theorem. The solution of the problem (1)-(6) is uniqueness by variables $y_{i}, i=1, \ldots, n$.

The uniqueness of meanings of variables $y_{i}$, $i=1, \ldots, n$ in optimal solution of the problem (1)-(6) for all numbers $\mathrm{k}$ guaranties single-valuedness of computed reliability indexes. 


\section{Computation of reliability indexes}

The modelling of random values is realized by next way. At first the number of working generators of every type is found. At next step the value of maximal generation is defined on the active generators. Additionally, random value of generated power is modelled for equipment with random generation. Procedure of random values modelling is detailed described in [8].

Adequacy analyzes is based on indexes which are computed as a result of many times estimation of random states of EPS. There are the most utilized indexes such as probability of non-deficit system work, mathematical expectation of power shortage and electricity supply coefficient [1]. The foreign indexes Loss of Load Expectation (LOLE) and Loss of Load Hours (LOLH) [17] are analogs of discussed indexes. For increasing quality of adequacy analysis it is possible to use special indexes which are took into account of peculiarities of researched energy system, for example, mathematical expectation of power generation on wind farms.

Let set of $\xi_{i}^{k}, \xi_{i}^{k}, €_{i j}^{k}$ is optimal solution of the problem (1)-(5), $k=1, \ldots, N, \quad i=1, \ldots, n$, $j=1, \ldots, n, \quad i \neq j$. The optimal value of power shortage in node $i, i=1, \ldots, n$, is defined by the formula

$$
d_{i}^{k}=\bar{y}_{i}^{k}-f_{i}^{k}, k=1, \ldots, N
$$

The state of EPS is deficit if the value

$$
d^{k}=\sum_{i=1}^{n} d_{i}^{k}
$$

is greater than zero. Let's numerate all deficit states of EPS and define of their common quantity as $H$. Then the probability of without shortage work of EPS are computed by next formula

$$
P=1-\frac{H}{N} \text {. }
$$

This index is corresponded to index LOLP.

Mathematical expectation of power shortage in nodes of energy system is computed by next rule

$$
M D_{i}=\sum_{j=1}^{H} \frac{d_{i}^{j}}{N}, i=1, \ldots, n .
$$

That's way mathematical expectation of power shortage in EPS is calculated by

$$
M D=\sum_{i=1}^{n} M D_{i} .
$$

For computing of mathematical expectation of power generation on a concrete type of equipment $l \in L$ we can use a next formula

$$
M X_{l}=\sum_{i=1}^{n} \frac{\tilde{x}_{i l}^{k}}{N}
$$

where $\tilde{x}_{i l}^{k}$ is optimal value of generation received on equipment type $l$ in node $i$ in $k$-state of EPS, $k=1, \ldots, N$. The given index can be useful in estimation of potential of generated power on wind power farms.

\section{Experimental research}

To illustrate and verify the developed methodology, consider the test scheme of EPS, consisting of 5 node and 5 links (Fig. 1).

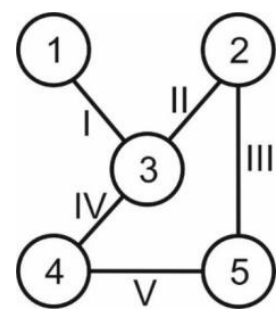

Fig. 1. The calculation scheme of the EPS

In Table. 1 are presented: characteristics of generating capacities (100 MW units with an accident rate of 0.045 p.u. were considered); the absolute maximum electrical load; in EPS nodes connected of 135-MW overhead lines with an integral accident rate of 0.011 p.u. / $100 \mathrm{~km}$ and a loss factor of $0.0000001661 \mathrm{MW} / 100 \mathrm{~km}$.

Table 1. Technical characteristics of the system.

\begin{tabular}{|c|c|c|c|c|c|c|}
\hline $\begin{array}{c}\text { Node } \\
\text { Number }\end{array}$ & 1 & 2 & 3 & 4 & 5 & System \\
\hline $\begin{array}{c}\text { Total } \\
\text { power in } \\
\text { the node, } \\
\text { MW }\end{array}$ & 500 & 1000 & 1400 & 1000 & 1200 & 5100 \\
\hline $\begin{array}{c}\text { Max } \\
\text { load, } \\
\text { MW }\end{array}$ & 700 & 700 & 1300 & 1000 & 1100 & 4800 \\
\hline $\begin{array}{c}\text { Overhead } \\
\text { line } \\
\text { number }\end{array}$ & I & II & III & IV & V & - \\
\hline $\begin{array}{c}\text { Number } \\
\text { of } \\
\text { overhead } \\
\text { line }\end{array}$ & 2 & 2 & 1 & 2 & 2 & - \\
\hline $\begin{array}{c}\text { Length, } \\
\text { km }\end{array}$ & 200 & 100 & 300 & 200 & 300 & - \\
\hline
\end{tabular}

In [8] the load graphs.

WT are located in node 1 . Total $20 \mathrm{WT}$ ENERCON E-82 E2 $2 \mathrm{MW}$ each. The accident rate of one WT is 0.9973 p.u. [18]. ES with a capacity of $2 \mathrm{MWh}$ were also located in the node number 1 . To simulate the wind speed, the statistics of meteorological observations obtained at station No. 20289 located on Russkiy Island were analyzed from 1967 to 1998 . A total of 84,891 observations were processed. In Table. 2 shows the histogram of frequencies.

Table 2. The histogram of the frequencies of the average daily velocity of a vector.

\begin{tabular}{|c|c|c|c|c|c|}
\hline $\begin{array}{c}\text { Group } \\
\text { interval, } \\
\mathbf{m} / \mathbf{s}\end{array}$ & {$[1,5)$} & {$[5,10)$} & {$[10,15)$} & {$[15,20)$} & {$[20,25]$} \\
\hline Freq-cy & 0,51152 & 0,40324 & 0,07523 & 0,00958 & 0,00001 \\
\hline
\end{tabular}


The treatment of long-term meteorological series in the meteorological station in question showed that during the autumn and winter seasons on the Russkiy Island, the direction of the wind predominates the northern direction (including the north-west and northeast), while in the spring-summer period the southern (including the southwestern and southeastern) Fig. 2.

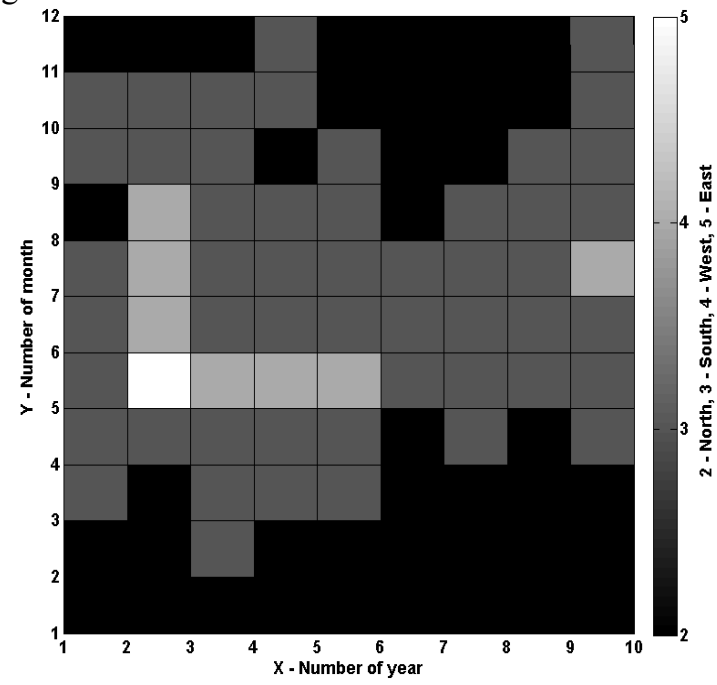

Fig. 2. The direction of the wind on the island Russkiy for 10 years the calculation period

At the same time, the average monthly wind speed exceeding $5 \mathrm{~m} / \mathrm{sec}$ is observed in the autumn-winter period with the predominant northern wind direction. In Fig. 3 shows the average monthly wind speed relative to 10 years of the calculation period.

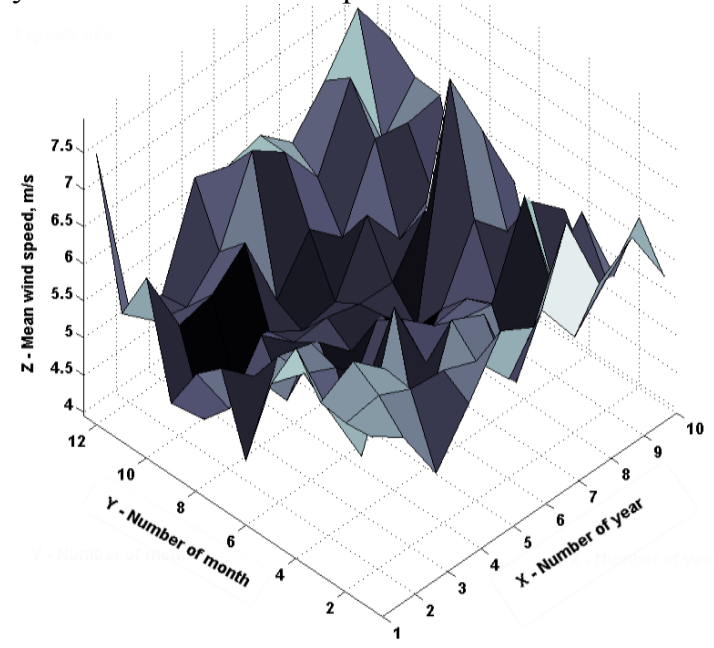

Fig. 3. Average monthly wind speed on the island Russkiy for 10 years of the calculation period

The simulated wind speed $v_{1}$ at a height of $10 \mathrm{~m}\left(h_{1}\right)$ to a height of $80 \mathrm{~m}\left(h_{2}\right)$ by formula [19]

$$
v_{2}=v_{1}\left(h_{1} / h_{2}\right)^{1 / 7}
$$

In Table. 3. The results of the evaluation of options for the development of EPS with different composition of
Table 3. Indicators of balance reliability for different equipment.

\begin{tabular}{|c|c|c|}
\hline $\begin{array}{c}\text { Number of WT, ES, } \\
\text { (installed capacity) }\end{array}$ & $\begin{array}{c}\text { Survival rate of } \\
\text { EPS }\end{array}$ & $\begin{array}{c}\text { Power shortage, } \\
\text { MW }\end{array}$ \\
\hline 10 WT (20 MW) & 0,994920 & 0,196116 \\
\hline 20 WT (40 MW) & 0,996140 & 0,136028 \\
\hline 30 WT (60 MW) & 0,996460 & 0,119281 \\
\hline $\begin{array}{c}\text { 10 WT (20 MW) } \\
\text { and ES (2 MWh) }\end{array}$ & 0,996600 & 0,107055 \\
\hline
\end{tabular}

The results show that the required reliability index $(0.996)$ is achieved by installing in node 1 or $20 \mathrm{WT}$ or 10 WT with ES with a total indicators obtained as a result of the input of $10 \mathrm{WT}$ with ES are higher than when installing $30 \mathrm{WT}$.

\section{Conclusion}

The paper presents a new methodology that allows estimating the balance reliability of EPS with WT and ES. Simulation of wind activity is carried out on the basis of the Monte Carlo method using frequency histograms of observed wind speeds. To estimate the power deficit of the generated random states of EPS, the methodology uses a modification of the power shortage estimation model with quadratic power losses in the overhead lines (the model of power shortage estimation with quadratic power losses in overhead lines). This model guarantees the uniqueness of the distribution of the power deficit at the nodes of the system and the unambiguity of the calculated reliability indicators. A new method for organizing computations for modeling the operation of ES is proposed. This feature greatly simplifies the computational process. The developed methodology of reliability analysis is tested on the test scheme of EPS, built on the basis of real technical data. To model the wind speed, we used a sample of long-term meteorological series, consisting of 84,891 observations. The results of calculations showed that the construction of WT and ES in EPS increases the level of reliability to the required regulatory values. At the same time, the best reliability was achieved by installing a $10 \mathrm{WT}$ and ES with a capacity of $2 \mathrm{MWh}$ in a deficit node. Further, in order to assess the feasibility of building new power plants using renewable energy, it is necessary to develop a methodology that takes into account the environmental component, the damage from under-supply of electricity, the cost of consumed fuel for energy production on traditional generation sources, capital costs.

\section{Acknowledgment}

This work is partially supported Russian Foundation for Basic Research, № 16-37-00333mol-a.

\section{References}

1. G.F. Kovalev, L.M. Lebedeva, Reliability of power systems (Novosibirsk, Nauka, 2015, 224 p.)

2. R. Billinton, R.N. Allan, Reliability Evaluation of Power Systems (Plenum Publishing, New York, 1996) 
3. Yu.Ya Chukreev, Model of reliability of electric power systems (Syktyvkar, Nauka, 1995, 176 p.)

4. Y.N. Rudenko, M.B. Chelcov Reliability and redundancy in power systems (Novosibirsk, Nauka, 1974, 263 p.)

5. G.F. Kovalev, L.M. Lebedeva, Complex models of optimizing the design state regimes in assessing the reliability of electric power systems (Irkutsk, ESI SB RAS, 2000, 73 p.)

6. D.S. Krupenev, S.M. Perzhabinskiy, J. Proceedings of the Russian Academy of Sciences. Power Engineering, 2, (2014)

7. D.S. Krupenev, S.M. Perzhabinskiy, J. Managing large systems, 54, (2015)

8. D.S. Krupenev, S.M. Perzhabinskiy, J. Proceedings of the Russian Academy of Sciences. Power Engineering, 2, (2017)

9. Ungjin Oh, Jaeseok Choi, Hag-hyeon Kim, IFACPapersOnLine 49-27, (2016)

10. Nian Shi, and Yi Luo, Energy Storage System Sizing Based on a Reliability Assessment of Power Systems Integrated with Wind Power, Sustainability, (2017)

11. Mabel M. C, Raj R. E, Fernandez E, Renewable and Sustainable Energy Reviews, 15, 2, (2011)

12. Billinton R, Huang D, IEEE Transactions On Power Systems, 26, 4, (2011)

13. Almutairi A, Ahmed M. H, Salama M. M. A, Electric Power Systems Research, 133, (2016)

14. Haquea A, Mandalb P, Mengc J, Negnevitsky M, International Journal of Sustainable Energy, 34 (2015)

15. Song J, Krishnamurthy V, Kwasinski A, Sharma R, IEEE Transactions on Sustainable Energy, 4, 2, (2013)

16. V.I. Zorkaltsev, S.M. Perzhabinskiy, J. Managing large systems, 30, 1, (2010)

17. Yu. YA. Chukreev, J. Proceedings of the Russian Academy of Sciences. Power Engineering, 6, (2012)

18. Echavarria E, Hahn B, van Bussel G.J, Tomiyama T, J. Sol. Energy Eng., 130, 3, (2008)

19. Nikolaev V.G, Ganaga S.V, Kudryashov Yu.I, National cadaster of Russia wind energy resources and methodology for their determination (Moscow, Atmograph, 2008. 584 p) 